\title{
The effect of unilateral surgical destruction of the cochlea on auditory sensitivity in the chinchilla
}

\author{
THOMAS L. BENNETT \\ Department of Psychology and Department of Physiology and Biophysics \\ and \\ R. JOHN MORGAN, PAULETTE MURPHY, and LUCIAN B. EDDY \\ Department of Physiology and Biophysics \\ Colorado State University, Fort Collins, Colorado 80523
}

\begin{abstract}
Since the monaural chinchilla has proven to be an excellent subject for research in audition, the present study sought to assess possible changes in the audibility curve resulting from surgically rendering the chinchilla monaural. Nine chinchillas were trained to respond to six pure tones by the method of instrumental avoidance conditioning, after which threshold testing was conducted using a modified method of limits. After establishing the audibility curve of the binaural chinchillas, sterile surgery was performed to destroy the left cochlea of each animal. When recovery from surgery was complete, the animals were retrained and retested. Auditory sensitivity for tones spaced at octave steps from .25 to $8.0 \mathrm{kHz}$ was found to decrease by a factor of $5 \mathrm{~dB}$ on the average as a result of the surgical procedure (range $=2.6-8.06 \mathrm{~dB}$ ).
\end{abstract}

In the past decade, the chinchilla has emerged as an excellent subject for behavioral investigations of auditory functioning. Initial work by Miller (1970) showed that the chinchilla can easily be trained to respond to tones by the method of instrumental avoidance conditioning. This technique has been shown to be effective for the measurement of pure-tone thresholds for chinchillas with normal or impaired hearing (Carder \& Miller, 1972; Henderson, 1969; Miller, 1970; Mills, 1973a, 1973b; Mills \& Talo, 1972), regardless of whether tested in noise or quiet (Miller, 1970; Mills, Talo, \& Gordon, 1973). These investigations have shown that the auditory world of the chinchilla is highly similar to that of humans (Miller, 1970).

Extensive studies on temporary, compound, or permanent threshold shifts, using the chinchilla as the experimental subject, have provided data pertinent to many questions about hearing loss resulting from exposure to noise (Carder \& Miller, 1972; Miller, Roghenberg, \& Eldredge, 1971; Mills \& Talo, 1972; Mills et al., 1973; Peters, 1965). When studies of this type are carried out, the animals are rendered surgically monaural to control or eliminate an acoustical interaction between ears (Miller, 1970; Strother, 1967). In view of the monaural chinchilla's use in these types of inquiry, it is important to obtain baseline data comparing binaural and monaural thresholds in these animals. In an early study addressed to this question, Miller (1970) measured audibility curves by behavioral methods in independent groups of chinchillas. He used two groups $(n=5)$ of binaural chinchillas and one group $(n=36)$ of monaural animals. It was found that one group of binaural animals had thresholds well below those of the monaural animals, while the other binaural group had thresholds above the monaural chinchillas. The basis for the differences between the two binaural groups was unclear.

The present study attempted to further examine binaural and monaural auditory sensitivity of the chinchilla. However, monaural and binaural audibility curves were assessed within the same animal. It was felt that this procedure would allow for better control of nonsurgical factors, such as differences in behavior of the animals, than did the independent-groups procedure.

\section{METHOD}

\section{Subjects}

A group of nine chinchillas, seven females and two males, were selected for this study. All were born and raised in a nearly soundproof concrete building with $30-\mathrm{cm}$ thick concrete walls and a $45-\mathrm{cm}$ thick roof. At the beginning of the study, the animals ranged in age from 14 to 26 months with a mean age of 18 months.

\section{Apparatus}

The equipment was modified after that used by Miller (1970). Audiometric training and testing were conducted with the animal in a single-walled sound-treated chamber (IAC 200 series), the inside of which was lined with $6.5-\mathrm{cm}$ egg-crate foam. Within the chamber, the major pieces of apparatus were the ceiling-mounted $30-\mathrm{cm}$ speaker, through which auditory signals were presented, and a suspended double-grill cage used for behavioral testing (46 cm long, $21 \mathrm{~cm}$ wide, $33 \mathrm{~cm}$ deep). A white plywood barrier $(8 \mathrm{~cm}$ high) divided the cage equally into two compartments. Attached to each side of the cage was a green light $(7 \mathrm{~W})$ and $6-\mathrm{V}$ door buzzer. The cage was constructed of $2.5-\mathrm{cm}$ wire mesh, with a conducting grid floor for administering the shock. The cage was suspended by wooden braces $60 \mathrm{~cm}$ below the speaker. Three white 7-W lights were attached to the wooden speaker support. No external light penetrated the sound 
chamber with the exception of a small slit $(2 \times 10 \mathrm{~cm})$ used by the experimenter for monitoring the animal's behavior.

The electronic equipment consisted of a Maico audiometer, Lafayette amplifier, Heathkit variable voltage regulator power supply, and a solid-state sequencer that was designed and built in our laboratory. The variable voltage regulator provided shock to either side of the cage at selected levels from 0 to $300 \mathrm{~V} \mathrm{dc}$ at currents of $0-2 \mathrm{~mA}$.

\section{Training}

Instrumental shock-avoidance conditioning similar to that described by Miller (1970) was used. The animals were trained to respond to tones by crossing the barrier prior to the onset of the buzzer (correct response). If the animal failed to respond to the tones, the buzzer automatically sounded $.75 \mathrm{sec}$ after the last tone pulse for a maximum of $2.5 \mathrm{sec}$. Electric shock paired temporarily to the buzzer was manually delivered in brief pulses until the animal crossed the barrier. Voltage settings of $100 \mathrm{~V} \mathrm{dc}$ were used. When the animal crossed the barrier, the sequence was manually terminated. The termination button also illuminated the green light on the "safe" side. This light remained on for about $5 \mathrm{sec}$ following a correct response. The intertrial intervals were random in duration, with a mean duration of $15 \mathrm{sec}$.

Prior to the first session of avoidance training, the animals were habituated to the apparatus for $30 \mathrm{~min}$. The trial sequence (Figure 1) was next introduced and pretraining began. For the pretraining period, the door to the chamber remained open, the intensity of the tones was high (about $70 \mathrm{~dB}$ ), and only one frequency $(1.0 \mathrm{kHz})$ was presented. Pretraining lasted no longer than 3-5 days (one 30-min session/day). Generally, acquisition of the avoidance response occurred rapidly and performance was in the range of $70 \%-100 \%$ at the end of pretraining.

Daily training trials were next initiated. Each.animal was given 10 trials at two different frequencies, for a total of 20 trials/day. The frequencies presented were in octave steps from .25 to $8.0 \mathrm{kHz}$ and were selected randomly. Initially, the intensity of the tone was $50 \mathrm{~dB}$ SPL. A minimum of 3 days was required to present the six frequencies using this method, after which the intensity was decreased $10 \mathrm{~dB}$. Reduction training continued until the animals were responding consistently at $10 \mathrm{db}$ SPL. The criterion for beginning binaural threshold tests was that an animal's response rate over the six test frequencies be $80 \%$ or better at intensities of $10 \mathrm{~dB}$.

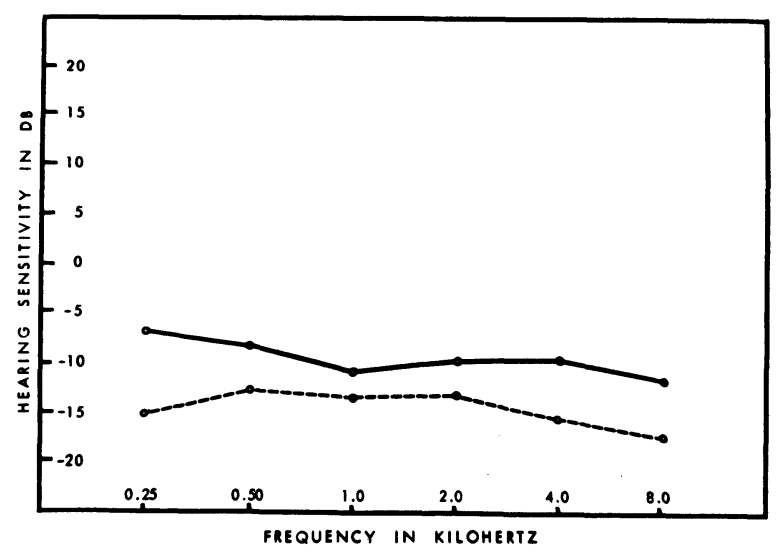

Figure 1. Hearing sensitivity in the monaural and binaural chinchilla. The solid line represents the average monaural audibility curve in octave steps from .25 to $8.0 \mathrm{kHz}$. The dotted line represents the mean binaural audibility curve for these frequency values.

\section{Threshold Testing}

The psychophysical method utilized in assessing auditory thresholds was a modified method of limits (Miller, Watson, \& Covell, 1963). On the first trial, the tone was presented at an intensity of $70 \mathrm{~dB}$ SPL. If the chinchilla responded correctly, the intensity was reduced $20 \mathrm{~dB}$ for the next trial. This sequence was followed until the chinchilla failed to respond correctly, after which the intensity of the tone was increased $10 \mathrm{~dB}$. If the animal again failed to respond, the level was increased $5 \mathrm{~dB}$; a $5-\mathrm{dB}$ reduction in intensity was made for a correct response. The threshold was defined as the average of the lowest sound level at which the animal responded and the highest level at which it failed to respond. Threshold measurements at each of the six test frequencies were determined daily for 5 consecutive days. A random order of frequency presentations was used each day. Results of the daily measurements were then averaged to establish a threshold. During threshold testing, the sequence was manually terminated prior to the onset of the buzzer if the animal failed to respond to a tone of an intensity below $30 \mathrm{~dB}$. Trials were repeated if the animal was inattentive or engaged in distracting activities (such as chewing on the wooden barrier).

\section{Surgery}

Each animal was rendered monaural by surgical destruction of the left cochlea employing the posterior approach to the cochlea described by Miller (1970). Surgery was performed under sterile conditions, with the animal anesthetized by ketamine hydrochloride $(30 \mathrm{mg} / \mathrm{kg})$ administered intramuscularly. The inside of the syringe used to administer the ketamine was pretreated with acepromazine maleate $(1 \mathrm{cc} / \mathrm{kg})$.

After the surgical field had been prepared, an incision along the posterior ridge of the auditory bulla was made. The round window of the cochlea was exposed by drilling a small hole in the posterior portion of the auditory bulla. A 45-deg-angle probe was inserted into the cochlea, puncturing the round window membrane. Lateral movements of the inserted probe were used to break the exposed cochlea from its base to its apex. The cochlear fluid and bone fragments were then aspirated, and the area was rinsed with sterile physiological saline solution. The destroyed cochlea was then packed with small pieces of gelfoam that had been dusted with tetracycline hydrochloride powder. A Zeiss variable-power operating microscope with an integral light source was used for the surgery. Cochlear destruction was histologically verified.

One month was allotted for recovery prior to retraining and retesting the animals. In our laboratory, we have noticed (unpublished) that some functional auditory adaptation, measured by behavioral audiometry, occurs within the month following surgery, prior to stabilizing.

\section{RESULTS AND DISCUSSION}

The average binaural and monaural audibility curves for the nine chinchillas are shown in Figure 1. Each data point is the mean of 45 threshold measurements. Table 1 compares average monaural and binaural threshold data. On the average, the binaural thresholds ranged from 2.6 to $8.09 \mathrm{~dB}$ lower than the monaural thresholds.

A randomized factorial design was used to evaluate the effect of surgical destruction of the left cochlea and the frequency parameters on behavioral thresholds. The analysis of variance is summarized in Table 2 . According to this analysis, a significant $(p<.01)$ decrease in auditory sensitivity occurred following surgical destruction of the left cochlea. These data compare favorably with information available from humans (Sivian \& 
Table 1

Mean Binaural and Monaural Thresholds and Sensitivity Change (SPL in dB, A Scale)

\begin{tabular}{lrrrrrr}
\hline & \multicolumn{6}{c}{ Frequency in Kilohertz } \\
\cline { 2 - 7 } & .25 & .50 & 1 & 2 & 4 & \multicolumn{1}{c}{8} \\
\hline Binaural & -15.02 & -12.67 & -13.40 & -13.04 & -15.40 & -17.15 \\
Monaural & -6.93 & -8.22 & -10.80 & -9.78 & -9.62 & -11.58 \\
$\begin{array}{l}\text { Auditory } \\
\text { Sensitivity } \\
\text { Change }\end{array}$ & 8.09 & 4.45 & 2.60 & 3.26 & 5.78 & 5.57 \\
\hline
\end{tabular}

Table 2

Analysis of Variance Summary Table

\begin{tabular}{lrrrr}
\hline \multicolumn{1}{c}{ Source } & \multicolumn{1}{c}{ SS } & df & MS & \multicolumn{1}{c}{ F } \\
\hline Blocks & 828.44 & 8 & 103.56 & 17.31 \\
Treatments & 923.78 & 11 & & \\
A & 661.65 & 1 & 661.65 & 110.64 \\
B & 171.79 & 5 & 34.36 & 5.76 \\
AB & 90.34 & 5 & 18.06 & 3.02 \\
Residual & 526.79 & 88 & 5.98 & 3.02 \\
Total & $2,279.01$ & 107 & & \\
\hline
\end{tabular}

Note-The effect of surgical destruction of the left cochlea (Treatment $A$ ) on hearing sensitivity was found to be significant at the $p<.01$ level. The variation in hearing sensitivity over the six frequencies (Treatment $B$ ) was not found to be significant, indicating that the audibility curve for the chinchilla, whether binaural or monaural, is relatively flat over this frequency range.

White, 1933) and the results obtained by Miller (1970), where he compared the audibility curves from one group of monaural chinchillas $(n=36)$ to two groups $(n=5 /$ group) of binaural animals. Taken as a whole, the binarual animals were slightly more sensitive than the monaural animals.

There are a variety of factors that could account for the reduction in hearing sensitivity found in the monaural chinchillas (Miller, 1970). Binaural animals may detect sound fields better than monaural animals due to a release from masking produced by either external or internal noise. An animal with two ears has a higher probability of having an ear in a pressure peak of the sound field. The two ears of a binaural animal may summate any auditory stimuli (Miller, 1970). The reduced sensitivity produced in the present study may be the result of one of many of these factors.

Both the monaural and binaural audibility curves were found to be lower than have previously been reported for the chinchilla. The average thresholds over the test frequencies were $-14.44 \mathrm{~dB}$ for binaural testing and $-9.48 \mathrm{~dB}$ during monaural testing of the chinchillas, indicating that the hearing sensitivity of the chinchilla is extremely acute. Threshold determinations (measured behaviorally) reflect not only the sensitivity of the receptor system, but nonsensory factors as well, such as training and performance. The fact that these animals were trained more extensively to respond to lower intensity tones $(10 \mathrm{~dB})$ and a wider range of test frequencies than in previous studies may account for this difference.

A final conclusion to be derived from this study is the reinforcement of the belief that the chinchilla is an excellent subject for this type of experimentation. The chinchillas were easy to train and displayed no resentful action toward the experimenter. It was surprising that the use of shock did not elicit hostile behavior. The cheerful, curious disposition of the chinchilla makes it a very enjoyable and suitable animal for behavioral experiments.

\section{REFERENCES}

CARDer, H. M., \& Miller, J. D. Temporary threshold shifts from prolonged exposure to noise. Journal of Speech and Hearing Research, 1972, 15, 603-623.

HeNDERSON, D. Temporal summation of acoustic signals by the chinchilla. Journal of the Acoustical Society of America, 1969, 46, 474-475.

Miller, J. D. Audibility curve of the chinchilla. Journal of the Acoustical Society of America, 1970, 48, 513-523.

Miller, J. D., Rothenberg, S. J., \& Eldredge, D. H. Preliminary observations of the effects of exposure to noise for seven days on the hearing and inner ear of the chinchilla. Journal of the Acoustical Society of America, 1971, 50, 1199-1203.

Miller, J. D., Watson, C. S., \& Covell, W. P. Deafening effects of noise on the cat. Acta Oto-Laryngologica, 1963, Supplement 176, $91 \mathrm{pp}$.

Milss, J. H. Temporary and permanent threshold shifts produced by nine-day exposure to noise. Journal of Speech and Hearing Research, 1973, 16, 426-438. (a)

Mills, J. H. Threshold shifts produced by exposure to noise in chinchillas with noise-induced hearing losses. Journal of Speech and Hearing Research, 1973, 16, 700-708. (b)

Mills, J. H., \& TALo, S. A. Temporary threshold shifts produced by exposure to high frequency noise. Journal of Speech and Hearing Research, 1972, 15, 624-631.

Mills, J. H., Talo, S. A., \& Gordon, G. S. Decay of temporary threshold shift in noise. Journal of Speech and Hearing Research, 1973, 16, 267-270.

Peters, E. N. Temporary shifts in auditory thresholds after exposure to noise. Journal of the Acoustical Society of America, 1965, 37, 831-833.

Sivian, L. J., \& White, S. D. On minimum audible sound fields. Journal of the Acoustical Society of America, 1933, 4, 288-321.

Strother, W. F. Hearing in the chinchilla (Chinchilla lanigera): I. Cochlear potentials. Journal of Auditory Research, 1967, 7, 145-155.

(Received for publication March 6, 1978.) 